\title{
Influence of chemical reaction, Soret and Dufour effects on heat and mass transfer of a binary fluid mixture in porous medium over a rotating disk
}

\author{
B.R.Sharma, Debozani Borgohain \\ Department of Mathematics, Dibrugarh University, Dibrugarh-786004, Assam, India
}

\begin{abstract}
Soret and Dufour effects on heat and mass transfer in steady flow over a rotating disk in porous medium are investigated. A set of coupled ordinary differential equations are obtained from the governing nonlinear partial differential equations which are then solved numerically by using MATLAB's built in solver bvp4c. The effects of material parameters like the porosity parameter, chemical reaction parameter, Dufour number, Soret number, Schmidt number and Prandtl number on temperature and concentration distributions are discussed through graphs. It is concluded that the porosity of the medium and the chemical reaction parameter play crucial role on the heat and mass transfer.
\end{abstract}

Keywords: Heat and Mass transfer; Porous Medium; Rotating disk; Dufour and Soret effects; chemical reaction.

\section{Introduction}

Researchers such as Nield and Bejan[1], Vafai[2] and Ingham and Pop[3, 4] have made comprehensive reviews in free convection heat and mass transfer through porous media. This area has many important applications in the field of large storage systems. Chemical reactions in the combined heat and mass transfer problems also have a great importance and therefore have attracted considerable interests of many researchers. Chemical reaction between a foreign mass and the fluid occurs in many industrial applications such as polymer production, the manufacturing of ceramics or glassware and so on.

Von Karman [5] introduced the flow due to an infinite rotating disk which is one of the classical problems in fluid mechanics. He formulated the problem in the steady state and then introduced similarity transformations to reduce the governing partial differential equations to ordinary differential equations. Asymptotic solutions for the steady hydrodynamic problem formulated by Von Karman were obtained by Cochran [6]. This gave the problem significant theoretical value and attracted many researchers attention. Benton [7] extended Cochran's work and solved the problem in transient state. Millsaps and Pohlhausen [8] considered the problem of heat transfer from a rotating disk at a constant temperature for a variety of Prandtl numbers in the steady state. The steady state heat transfer from a rotating disk maintained at a constant temperature to fluids at any Prandtl number was studied by Sparrow and Gregg [9]. The flow above a rotating disk in the presence of an external uniform magnetic field was studied by Attia et.al. [10-12]. Stuart [13], Kuiken [14], Ockendon [15], and Attia [16-18] investigated the effect of uniform suction or injection through a rotating porous disk on the steady hydrodynamic or hydromagnetic flow induced by the disk.

H. A. Attia [19] investigated numerically the steady flow and heat transfer of an incompressible viscous fluid in a porous medium due to a rotating disk neglecting Soret and Dufour effects. This paper is an extension of the above mentioned paper in which Soret and Dufour effects in presence of chemical reaction on heat and mass transfer are investigated.

\section{Mathematical Formulation}

Consider a three dimensional laminar, steady flow of a viscous incompressible binary fluid mixture in a porous medium over a rotating disk of infinite extent. It is considered that the disk lie in the plane $\mathrm{z}=0$ and the motion of the fluid is due to its rotation about an axis perpendicular to its plane with constant angular speed $\omega$. The ambient temperature far away from the surface of the disk is $T_{\infty}$ and the surface of the disk is maintained at constant temperature $T_{W}$ such that $T_{W}>T_{\infty}$. The concentration of the fluid at the surface is $C_{W}$ and far away from the disk is $\mathrm{C}_{\infty}$ such that $\mathrm{C}_{\mathrm{W}}>\mathrm{C}_{\infty}$. It is assumed that the physical properties of the fluid mixture are constant. It is also assumed that first order chemical reaction takes place in the flow of the binary fluid mixture.

Under the above assumptions, the governing equations describing the conservation of mass, momentum, energy and concentration in cylindrical polar coordinates $(r, \emptyset, z)$ can be written as follows:

$\frac{\partial \mathrm{u}}{\partial \mathrm{r}}+\frac{\mathrm{u}}{\mathrm{r}}+\frac{\partial \mathrm{w}}{\partial \mathrm{z}}=0$

$\rho\left(u \frac{\partial u}{\partial r}+w \frac{\partial u}{\partial z}-\frac{v^{2}}{r}\right)+\frac{\partial p}{\partial r}=\mu\left(\frac{\partial^{2} u}{\partial r^{2}}+\frac{1}{r} \frac{\partial u}{\partial r}-\frac{u}{r^{2}}+\frac{\partial^{2} u}{\partial z^{2}}\right)-\frac{\mu}{K} u$ 
$\rho\left(\mathrm{u} \frac{\partial \mathrm{v}}{\partial \mathrm{r}}+\mathrm{w} \frac{\partial \mathrm{v}}{\partial \mathrm{z}}+\frac{\mathrm{uv}}{\mathrm{r}}\right)=\mu\left(\frac{\partial^{2} \mathrm{v}}{\partial \mathrm{r}^{2}}+\frac{1}{\mathrm{r}} \frac{\partial \mathrm{v}}{\partial \mathrm{r}}-\frac{\mathrm{v}}{\mathrm{r}^{2}}+\frac{\partial^{2} \mathrm{v}}{\partial \mathrm{z}^{2}}\right)-\frac{\mu}{\mathrm{K}} \mathrm{v}$

$\rho\left(\mathrm{u} \frac{\partial \mathrm{w}}{\partial \mathrm{r}}+\mathrm{w} \frac{\partial \mathrm{w}}{\partial \mathrm{z}}\right)+\frac{\partial \mathrm{p}}{\partial \mathrm{z}}=\mu\left(\frac{\partial^{2} \mathrm{w}}{\partial \mathrm{r}^{2}}+\frac{1}{\mathrm{r}} \frac{\partial \mathrm{w}}{\partial \mathrm{r}}-\frac{\mathrm{w}}{\mathrm{r}^{2}}+\frac{\partial^{2} \mathrm{w}}{\partial \mathrm{z}^{2}}\right)-\frac{\mu}{\mathrm{K}} \mathrm{w}$

$\rho \mathrm{C}_{\mathrm{p}}\left(\mathrm{u} \frac{\partial \mathrm{T}}{\partial \mathrm{r}}+\mathrm{w} \frac{\partial \mathrm{T}}{\partial \mathrm{z}}\right)=\alpha\left(\frac{\partial^{2} \mathrm{~T}}{\partial \mathrm{z}^{2}}+\frac{\partial^{2} \mathrm{~T}}{\partial \mathrm{r}^{2}}+\frac{1}{\mathrm{r}} \frac{\partial \mathrm{T}}{\partial \mathrm{r}}\right)+\frac{\rho \mathrm{D}_{\mathrm{m}} \mathrm{K}_{\mathrm{T}}}{\mathrm{C}_{\mathrm{s}}}\left(\frac{\partial^{2} \mathrm{C}}{\partial \mathrm{z}^{2}}+\frac{\partial^{2} \mathrm{C}}{\partial \mathrm{r}^{2}}+\frac{1}{\mathrm{r}} \frac{\partial \mathrm{C}}{\partial \mathrm{r}}\right)$

$\mathrm{u} \frac{\partial \mathrm{C}}{\partial \mathrm{r}}+\mathrm{w} \frac{\partial \mathrm{C}}{\partial \mathrm{z}}=\frac{\mathrm{D}_{\mathrm{m}} \mathrm{K}_{\mathrm{T}}}{\mathrm{T}_{\mathrm{m}}}\left(\frac{\partial^{2} \mathrm{~T}}{\partial \mathrm{z}^{2}}+\frac{\partial^{2} \mathrm{~T}}{\partial \mathrm{r}^{2}}+\frac{1}{\mathrm{r}} \frac{\partial \mathrm{T}}{\partial \mathrm{r}}\right)+\mathrm{D}_{\mathrm{m}}\left(\frac{\partial^{2} \mathrm{C}}{\partial \mathrm{z}^{2}}+\frac{\partial^{2} \mathrm{C}}{\partial \mathrm{r}^{2}}+\frac{1}{\mathrm{r}} \frac{\partial \mathrm{C}}{\partial \mathrm{r}}\right)-\mathrm{k}_{1}\left(\mathrm{C}-\mathrm{C}_{\infty}\right)$

where $\mathrm{u}, \mathrm{v}, \mathrm{w}$ are velocity components in the directions of increasing $r, \emptyset, \mathrm{z}$ respectively, $\mathrm{p}$ is the pressure, $\mu$ is the coefficient of viscosity, $\rho$ is the density of the fluid mixture, $\mathrm{K}$ is permeability of porous medium, $K_{T}$ is the thermal diffusion ratio, $\alpha$ is the thermal diffusivity, $D_{m}$ is the mass diffusivity, $T$ is temperature, $C$ is concentration, $C_{p}$ is the specific heat capacity, $C_{S}$ is the concentration susceptibility, $T_{m}$ is the mean fluid temperature and $\mathrm{k}_{1}$ is the dimensional chemical reaction parameter.

The boundary conditions are

$\mathrm{u}=0, \mathrm{v}=\mathrm{r} \omega, \mathrm{w}=0, \mathrm{~T}=\mathrm{T}_{\mathrm{W}}, \mathrm{C}=\mathrm{C}_{\mathrm{W}}$ at $\mathrm{z}=0$

$\mathrm{u} \rightarrow 0, \mathrm{v} \rightarrow 0, \mathrm{~T} \rightarrow \mathrm{T}_{\infty}, \mathrm{C} \rightarrow \mathrm{C}_{\infty}$ as $\mathrm{z} \rightarrow \infty$

\}

We introduce Von Karman transformations

$\mathrm{u}=\mathrm{r} \omega \mathrm{F}, \mathrm{v}=\mathrm{r} \omega \mathrm{G}, \mathrm{w}=\sqrt{\omega \nu} \mathrm{H}, \mathrm{z}=\sqrt{\nu / \omega} \zeta, \mathrm{p}-\mathrm{p}_{\infty}=-\rho \nu \omega \mathrm{P}$

$\theta=\frac{\mathrm{T}-\mathrm{T}_{\infty}}{\mathrm{T}_{\mathrm{W}}-\mathrm{T}_{\infty}}, \quad \varnothing=\frac{\mathrm{C}-\mathrm{C}_{\infty}}{\mathrm{C}_{\mathrm{W}}-\mathrm{C}_{\infty}}$

where $\zeta$ is the non-dimensional distance measured along the axis of rotation. $\mathrm{F}, \mathrm{G}, \mathrm{H}$ and $\mathrm{P}$ are the nondimensional functions of $\zeta, v$ is the kinematic viscosity of the fluid given by $v=\mu / \rho$.

Introducing the relation (8) - (9) into the equations (1) - (6), we obtain the following system of ordinary coupled differential equations:

$\mathrm{H}^{\prime}+2 \mathrm{~F}=0$

$\mathrm{F}^{/ /}-\mathrm{HF}^{/}-\mathrm{F}^{2}+\mathrm{G}^{2}-\mathrm{D}_{\mathrm{a}} \mathrm{F}=0$

$\mathrm{G}^{/ /}-\mathrm{HG}^{/}-2 \mathrm{FG}-\mathrm{D}_{\mathrm{a}} \mathrm{G}=0$

$\mathrm{H}^{/ /}-\mathrm{HH}^{/}+\mathrm{P}^{/}-\mathrm{D}_{\mathrm{a}} \mathrm{H}=0$

$\frac{1}{\operatorname{Pr}} \theta^{/ /}-\mathrm{H} \theta^{/}+\mathrm{D}_{\mathrm{f}} \phi / /=0$

$\frac{1}{\mathrm{Sc}} \emptyset / /-\mathrm{H} \emptyset /+\mathrm{Sr} \theta / /-\gamma \emptyset=0$

where $D_{a}$ is porosity parameter, Pr is Prandtl number, Sc is Schmidt number, $D_{f}$ is Dufour number, Sr is Soret number and $\gamma$ is dimensionless chemical reaction parameter and are defined as:

$\mathrm{D}_{\mathrm{a}}=\frac{v}{\mathrm{~K} \omega}, \operatorname{Pr}=\frac{\mu \mathrm{C}_{\mathrm{p}}}{\alpha}, \mathrm{Sc}=\frac{v}{\mathrm{D}_{\mathrm{m}}}, \mathrm{D}_{\mathrm{f}}=\frac{\mathrm{D}_{\mathrm{m}} \mathrm{K}_{\mathrm{T}}}{\mathrm{C}_{\mathrm{S}} \mathrm{C}_{\mathrm{P}}} \frac{\left(\mathrm{C}_{\mathrm{W}}-\mathrm{C}_{\infty}\right)}{v\left(\mathrm{~T}_{\mathrm{W}}-\mathrm{T}_{\infty}\right)}, \mathrm{Sr}=\frac{\mathrm{D}_{\mathrm{m}} \mathrm{K}_{\mathrm{T}}\left(\mathrm{T}_{\mathrm{W}}-\mathrm{T}_{\infty}\right)}{v \mathrm{~T}_{\mathrm{W}}\left(\mathrm{C}_{\mathrm{W}}-\mathrm{C}_{\infty}\right)}, \gamma=\frac{\mathrm{k}_{1}}{\omega}$

In equations (10) - (15) a dash denotes diffentiation with respect to $\zeta$.

The boundary conditions (7) are now transformed to

$\mathrm{F}=0, \mathrm{G}=1, \mathrm{H}=0, \theta=1, \varnothing=1 \quad$ at $\quad \zeta=0$

$\mathrm{F} \rightarrow 0, \mathrm{G} \rightarrow 0, \theta \rightarrow 0, \varnothing \rightarrow 0 \quad$ as $\quad \zeta \rightarrow \infty$

The system of ordinary coupled differential equations (10), (11), (12), (14) and (15) under the boundary conditions (17) have been solved numerically by using MATLAB's built in solver bvp4c. Equation (13) can be used to solve the pressure distribution if required.

\section{Results And Discussion}

Numerical calculations have been carried out for various values of the parameters $D_{f}, \gamma, D_{a}, \operatorname{Pr}, S c$ and Sr. Six cases are considered:

Case I : $\mathrm{D}_{\mathrm{f}}=(0,0.15,0.2,0.3), \gamma=0.2, \mathrm{D}_{\mathrm{a}}=0.5, \mathrm{Pr}=7, \mathrm{Sc}=1.6, \mathrm{Sr}=0.1$

Case II : $\mathrm{D}_{\mathrm{f}}=0.15, \gamma=(0.2,0.6,1,2), \mathrm{D}_{\mathrm{a}}=0.5, \mathrm{Pr}=7, \mathrm{Sc}=1.6, \mathrm{Sr}=0.1$

Case III : $\mathrm{D}_{\mathrm{f}}=0.15, \gamma=0.2, \mathrm{D}_{\mathrm{a}}=(0.5,0.7,0.9,1.2), \mathrm{Pr}=7, \mathrm{Sc}=1.6, \mathrm{Sr}=0.1$

Case IV : $\mathrm{D}_{\mathrm{f}}=0.15, \gamma=0.2, \mathrm{D}_{\mathrm{a}}=0.5, \mathrm{Pr}=(2,4,7,13.2), \mathrm{Sc}=1.6, \mathrm{Sr}=0.1$

Case V : $\mathrm{D}_{\mathrm{f}}=0.15, \gamma=0.2, \mathrm{D}_{\mathrm{a}}=0.5, \mathrm{Pr}=7, \mathrm{Sc}=(1.1,1.6,2.2,3), \mathrm{Sr}=0.1$

Case VI : $\mathrm{D}_{\mathrm{f}}=0.15, \gamma=0.2, \mathrm{D}_{\mathrm{a}}=0.5, \mathrm{Pr}=7, \mathrm{Sc}=1.6, \mathrm{Sr}=(0.01,0.1,0.2,0.3)$

The numerical results for temperature and concentration profiles are displayed in Figures (1) - (6).

Case I:

Figures 1 (a)-(b) exhibit temperature and concentration profile for various values of $\mathrm{D}_{\mathrm{f}}$. It is observed that the temperature and concentration decreases exponentially from their maximum values at the surface to their minimum values at the end of the boundary layer. It is also noticed that with an increase in the values of Dufour number $D_{f}$, the temperature of the binary fluid mixture increases sharply while the concentration of the 
binary fluid mixture decreases slightly. Hence the effect of $\mathrm{D}_{\mathrm{f}}$ on temperature distribution is more than that of concentration distribution.

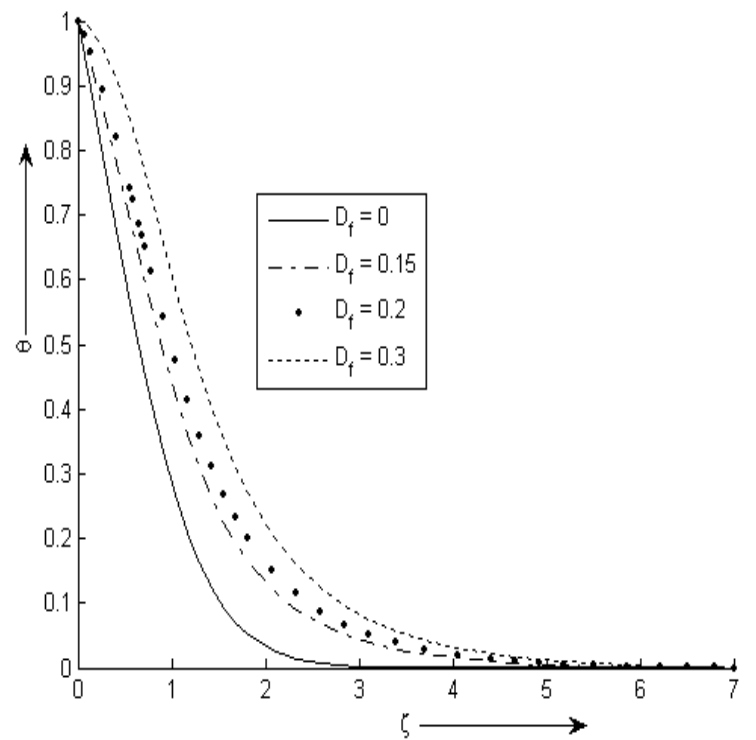

(a)

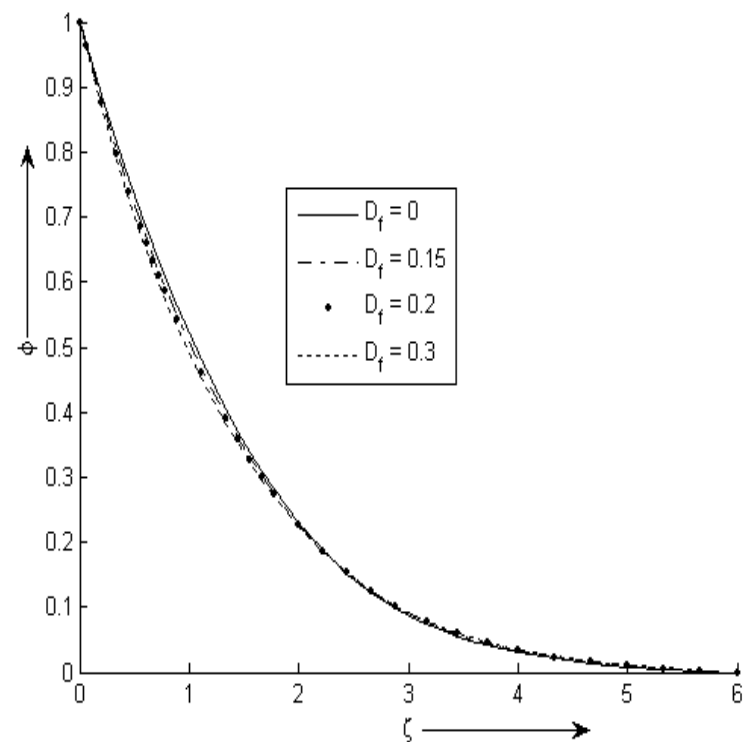

(b)

Fig. 1 : Effect of Dufour number $\mathrm{D}_{\mathrm{f}}$ on (a) the temperature profiles, (b) the concentration profiles

\section{Case II:}

Figures 2 (a)-(b) exhibit temperature and concentration profile for various values of $\gamma$. It is noticed that the temperature and concentration decreases exponentially from their maximum values at the surface to their minimum values at the end of the boundary layer. It is also observed that with an increase in the values of the chemical reaction parameter $\gamma$, the temperature of the binary fluid mixture increases while the concentration of the binary fluid mixture decreases sharply.

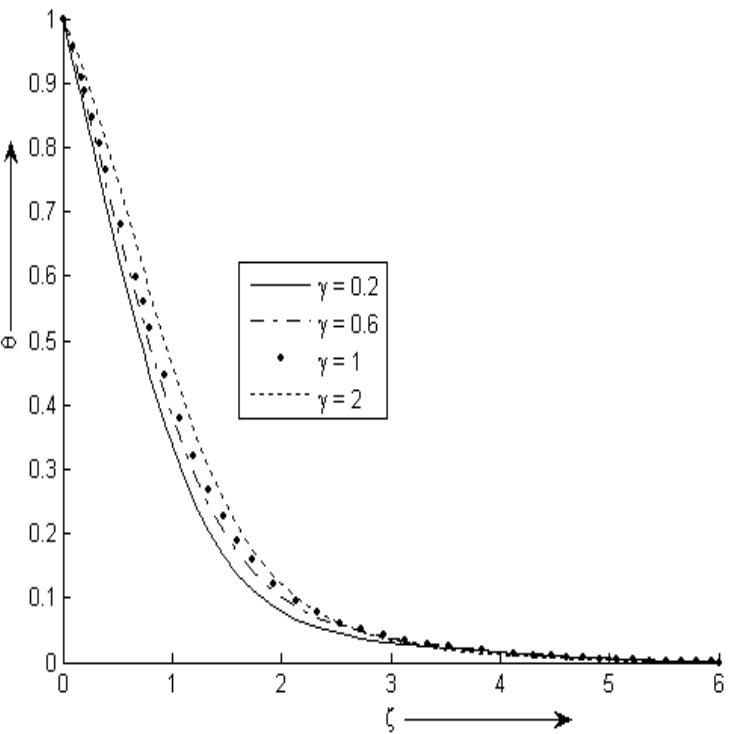

(a)

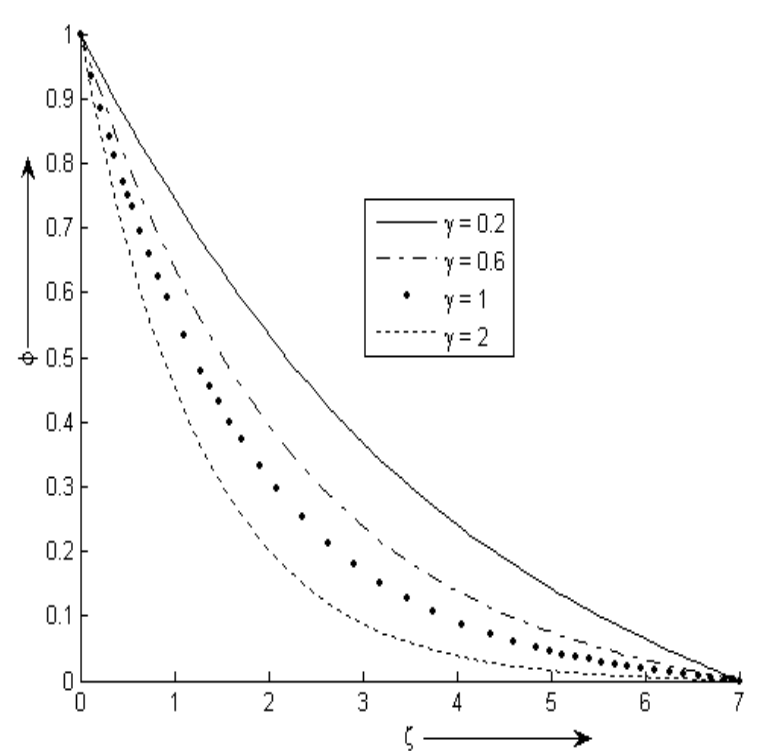

(b)

Fig. 2 : Effect of Chemical reaction parameter $\gamma$ on (a) the temperature profiles, (b) the concentration profiles

\section{Case III:}

Figures 3 (a)-(b) depict temperature and concentration profile for various values of $\mathrm{D}_{\mathrm{a}}$. It is noticed that there is an exponential decrease in the temperature and concentration profiles from their maximum values at the surface to their minimum values at the end of the boundary layer. It is also observed that with an increase in the values of the porosity parameter $\mathrm{D}_{\mathrm{a}}$, the temperature of the binary fluid mixture increases sharply and the concentration of the rarer and lighter components in the fluid increases. 


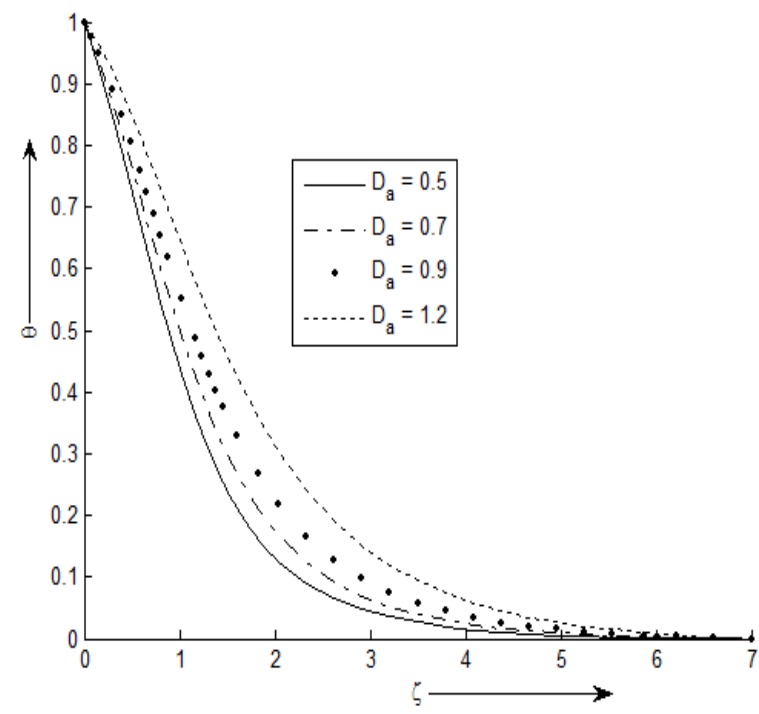

(a)

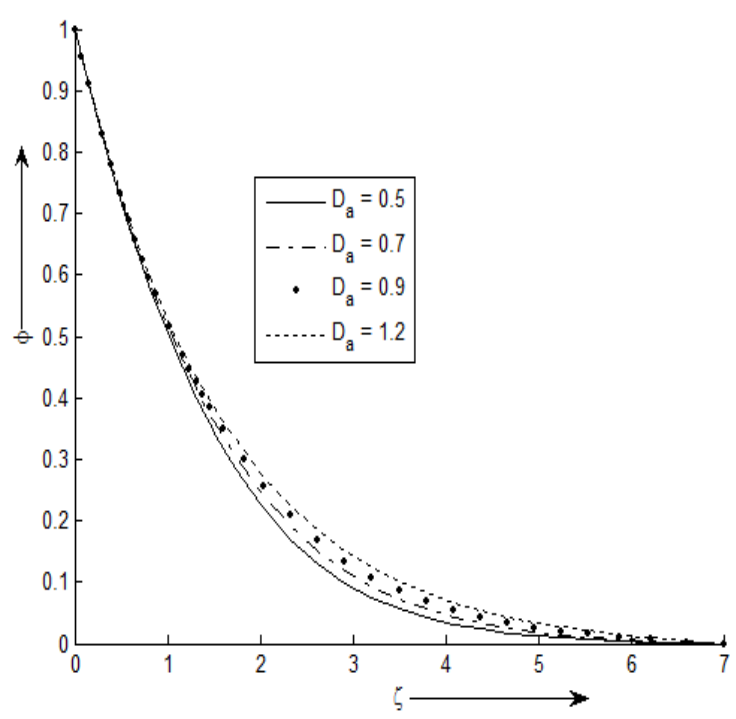

(b)

Fig. 3 : Effect of Porosity parameter $D_{a}$ on (a) the temperature profiles, (b) the concentration profiles

\section{Case IV:}

Figures 4 (a)-(b) exhibit temperature and concentration profile for various values of Pr. It is observed that the temperature and concentration decreases exponentially from their maximum values at the surface to their minimum values at the end of the boundary layer. It is also noticed that with an increase in the values of the Prandtl number Pr, the temperature of the binary fluid mixture decreases sharply while the concentration of the binary fluid mixture increases slightly.

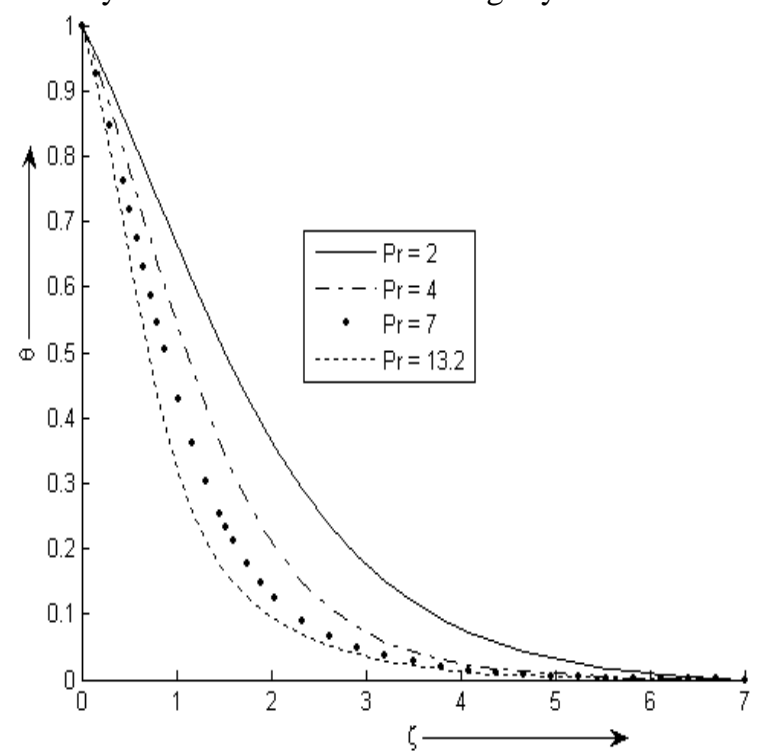

(a)

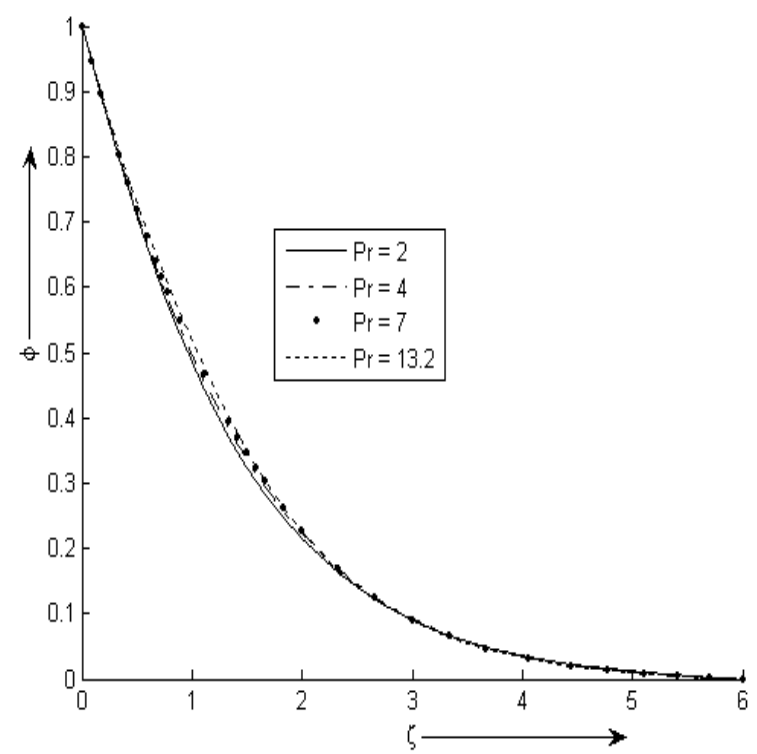

(b)

Fig. 4 : Effect of Prandtl number Pr on (a) the temperature profiles, (b) the concentration profiles

\section{Case V:}

Figures 5 (a) - (b) exhibit temperature and concentration profile for various values of Sc. It is noticed that the temperature and concentration decreases exponentially from their maximum values at the surface to their minimum values at the end of the boundary layer. It is also observed that the temperature of the binary fluid mixture increases slightly near the surface with the increase in the values of Schmidt number Sc but gets reversed after $\zeta=2$ i.e. away from the surface while the concentration of the rarer and lighter components in the fluid decreases sharply with the increase in the values of Sc. 


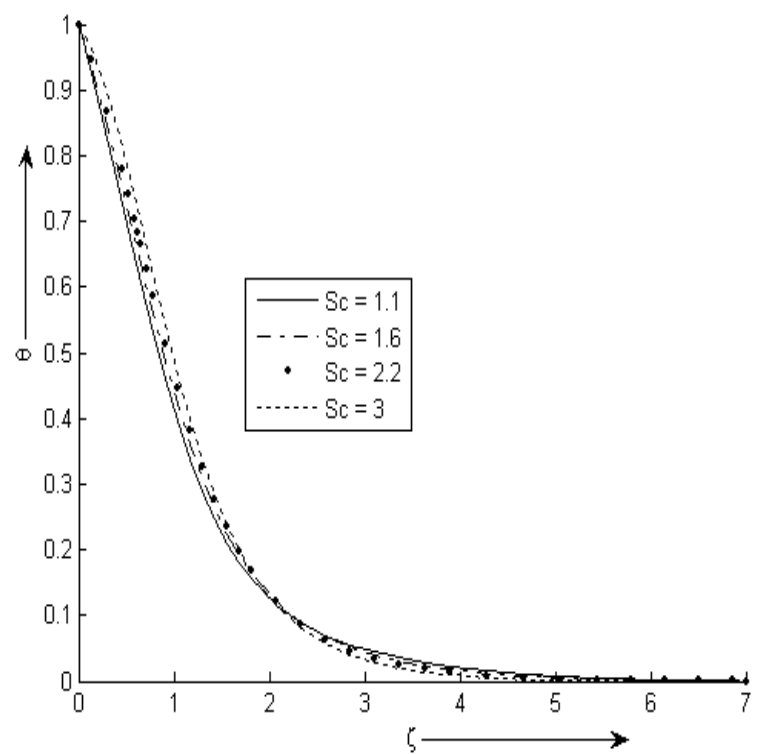

(a)

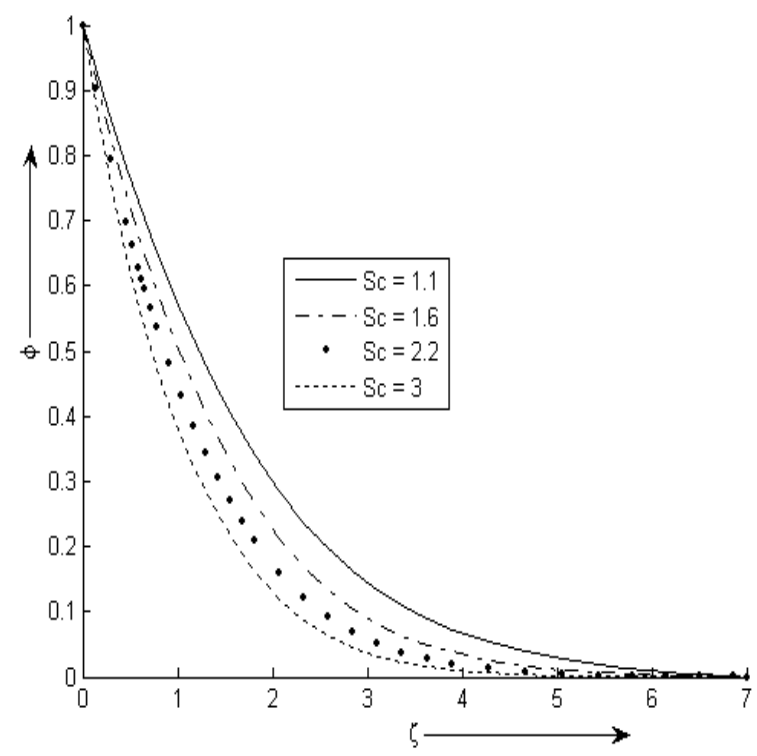

(b)

Fig. 5 : Effect of Schmidt number Sc on (a) the temperature profiles, (b) the concentration profiles

\section{Case VI:}

Figures 6 (a) - (b) exhibit temperature and concentration profile for various values of Sr. It is noticed that the temperature and concentration decreases exponentially from their maximum values at the surface to their minimum values at the end of the boundary layer. It is also observed that the temperature of the binary fluid mixture decreases slightly near the surface with the increase in the values of Soret number Sr but gets reversed after $\zeta=2$ i.e. away from the surface while the concentration of the rarer and lighter components in the fluid increases sharply with the increase in the values of $\mathrm{Sr}$.

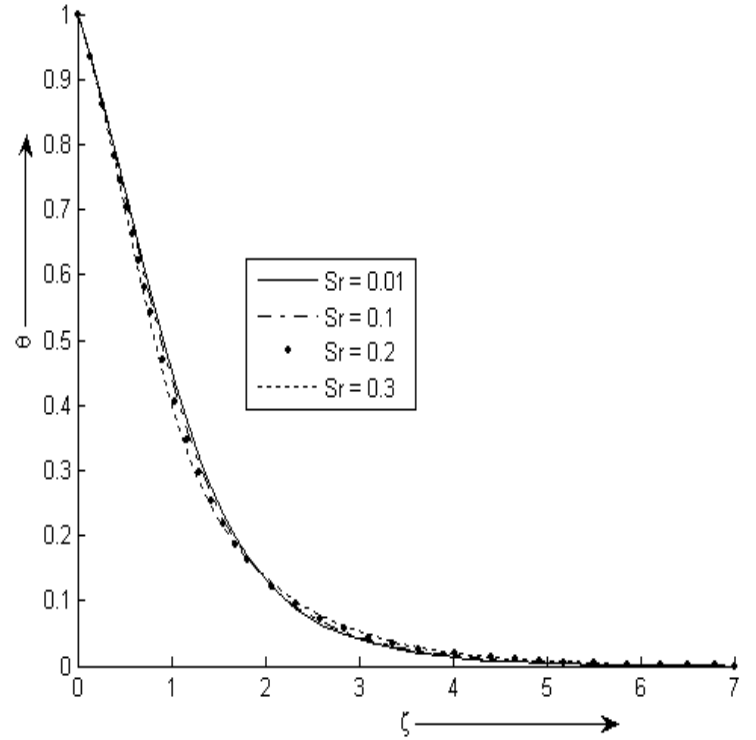

(a)

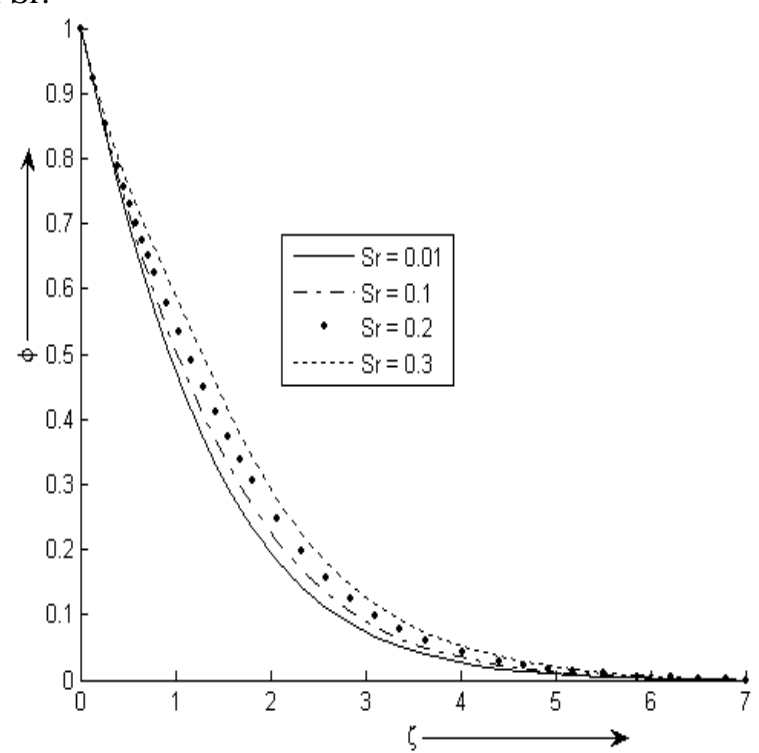

(b)

Fig. 6 : Effect of Soret number Sr on (a) the temperature profiles, (b) the concentration profiles

\section{Conclusion}

From above analysis it can be concluded that the temperature of the binary fluid mixture decreases with the increase of Prandtl number whereas increases with the increase of Dufour number, dimensionless chemical reaction parameter and porosity parameter. Also the temperature increases with the increase in Schmidt number and decreases with the increase in Soret number near the surface but the result gets reversed away from the surface. Concentration of the rarer and lighter component of the binary fluid mixture increases with the increase of porosity parameter, Prandtl number and Soret number whereas decreases with the increase of Dufour number, dimensionless chemical reaction parameter and Schmidt number. It is hoped that the present work will serve as a motivation for future experimental work which seems to be lacking at the present time. 


\section{References}

[1]. Nield, D.A. and Bejan, A. (2006). Convection in Porous Media, $3^{\text {rd }}$ edition, Springer, New York.

[2]. Vafai, K. (2000). Handbook of Porous Media, Marcel Dekker, New York.

[3]. Ingham, D.B. and Pop, I. (1998). Transport Phenomena in Porous Media I, Pergamon, Oxford.

[4]. Ingham, D.B. and Pop, I. (2002). Transport Phenomena in Porous Media II, Pergamon, Oxford.

[5]. Von Karman, T.(1921). Uber laminare und turbulente reibung, ZAMM, 1(4), pp.233-235.

[6]. Cochran, W.G. (1934). The flow due to a rotating disk, P. Camb. Philos. Soc., 30(3), pp.365-375.

[7]. Benton, E.R. (1966). On the flow due to a rotating disk, J. Fluid Mech., 24(4), pp.781-800.

[8]. Millsaps, K. and Pohlhausen, K. (1952). Heat transfer by laminar flow from a rotating disk, J. Aeronaut. Sci., 19, pp.120-126.

[9]. Sparrow, E.M. and Gregg, J.L. (1960). Mass transfer, flow, and heat transfer about a rotating disk, ASME J. Heat Transfer, pp.294302, Nov.

[10]. El-Mistikawy, T.M.A. and Attia, H.A. (1990). The rotating disk flow in the presence of strong magnetic field, Proc. 3rd Int. Congress of Fluid Mechanics, Cairo, Egypt, vol. 3, pp. 1211-1222, Jan. 2-4.

[11]. El-Mistikawy, T.M.A., Attia, H.A., and Megahed, A.A. (1991). The rotating disk flow in the presence of weak magnetic field, Proc. 4th Conference on Theoretical and Applied Mechanics, Cairo, Egypt, pp. 69-82, Nov. 5-7.

[12]. Aboul-Hassan, A.L. and Attia, H.A. (1997). The flow due to a rotating disk with Hall effect, Physics Letters A, vol. 228, pp. 286290 .

[13]. Stuart, J.T. (1954). On the effects of uniform suction on the steady flow due to a rotating disk, Q. J. Mech. Appl. Math., 7, pp.446457.

[14]. Kuiken, H.K. (1971). The effect of normal blowing on the flow near a rotating disk of infinite extent, J. Fluid Mech., 47(4), pp.789798.

[15]. Ockendon, H. (1972). An asymptotic solution for steady flow above an infinite rotating disk with suction, Q. J. Mech. Appl. Math., 25, pp.291-301.

[16]. Attia, H.A. (1998). Unsteady MHD flow near a rotating porous disk with uniform suction or injection, Fluid Dyn. Res., 23, pp.283290 .

[17]. Attia, H.A. , Aboul-Hassan, A.L. (2001). Effect of Hall current on the unsteady MHD flow due to a rotating disk with uniform suction or injection, Appl. Math. Model., 25(12), pp.1089-1098.

[18]. Attia, H.A. (2002). On the effectiveness of uniform suction-injection on the unsteady flow due to a rotating disk with heat transfer, Int. Commun. Heat Mass, 29(5), pp.653-661.

[19]. Attia, H.A. (2009). Steady Flow over a Rotating Disk in Porous Medium with Heat Transfer Nonlinear Analysis, Modelling and Control, Vol. 14, No.1, pp.21-26. 